\title{
Impressum, Vol. 7, No. 6, 1984
}

\section{S. Karger}

Verlag für Medizin und Naturwissenschaften GmbH, Postfach 1724, D-8034 Germering

\section{Wissenschaftlicher Beirat}

H. W. Bauer, München; H. Denck, Wien; V. Diehl, Köln; F. Douwes, Göttingen; P. Drings, Heidelberg; S. Eckhardt, Budapest; H. Ehrhart, München; W. M. Gallmeier, Nürnberg; H. Gerhartz, Berlin; E. H. Graul, Marburg/Lahn; R. Gross, Köln; H. Heimpel, Ulm; K. P. Hellriegel, Berlin; Ch. Herfarth, Ulm; J. H. Holzner, Wien; R. Hünig, Basel; W. Hunstein, Heidelberg; H. O. Klein, Köln; H. Löffler, Kiel; U. Mohr, Hannover; F.-L. Müller, Bonn; K. Munk, Heidelberg; G. P. Murphy, Buffalo (N.Y.); G. A. Nagel, Göttingen; A. Pfleiderer, Freiburg; Mildred Scheel, Bonn; E. Scherer, Essen; S. Seeber, Essen; H. J. Senn, St. Gallen; A. Stacher, Wien; St. Tanneberger, Berlin/DDR; W. Vahlensieck, Bonn; H. D. Waller, Tubingen; M. Wannen-macher, Freiburg; W. Wilmanns, München; K. Wilms, Würzburg; K. zum Winkel, Heidelberg; H. Wrba, Wien.

Verantwortliche Schriftleitung z. Zt. S. Eckhardt, Budapest J. H. Holzner, Wien G. A. Nagel, Göttingen

Die Zeitschrift erscheint zweimonatlich; pro Jahr erscheint 1 Band zu je 6 Heften.

Bezugspreis für Jahrgang 7, 1984 DM 138,-/öS 966,-/SFr. 124,-. 1 Einzelheft kostet DM 26,-/öS 182,-/SFr. 25,-, einschließlich MwSt., zuzüglich Postgebühren.

Der Abonnementspreis ist im voraus zahlbar. Die Lieferung der Zeitschrift läuft weiter, wenn sie nicht spätestens 4 Wochen vor Abschluß eines Bandes abbestellt wird.

Abonnementsbestellungen können bei jeder Buchhandlung, bei der Post oder direkt beim Verlag aufgegeben werden: Bundesrepublik Deutschlandl Österreich: S. Karger GmbH, Postfach 1724, D-8034 Germering/München, Tel.: (089) 844021, Telex: 524865 D, Postscheckkonto: München 40080-807 Schweiz: S. Karger AG, Allschwilerstr. 10, Postfach, CH-4009 Basel, Tel.: (061) 390880, Telex $62652 \mathrm{CH}$ Anzeigen

S. Karger Verlag für Medizin und Naturwissenschaften $\mathrm{GmbH}$. Postfach 1724, D-8034 Germering, Telefon (089) 844021.

Gültig ist die Preisliste Nr. 4 vom 1.10.1982 (Einkleber aui

Anfrage).

Für den Inhalt außerhalb des redaktionellen Teiles (insbesonden Anzeigen, Industrieinformationen, Pressezitate und Kongreßinfor-mationen) übernehmen Schriftleitung, Beirat und Verlag keine Ge währ.

Eine Markenbezeichnung kann warenzeichenrechtlich geschütz sein, auch wenn bei ihrer Verwendung in dieser Zeitschrift dai Zeichen ${ }^{\circledR}$ oder ein anderer Hinweis auf etwa bestehende Schutz rechte fehlen sollte.

Alle Rechte, insbesondere das Recht der Vervielfältigung unc Mikrokopie sowie der Übersetzung, vorbehalten. Nachdruck, aucl auszugsweise, nur mit Genehmigung des Verlages. 
(C) Copyright 1984 by S. Karger Verlag für Medizin und Natur wissenschaften GmbH, Postfach 1724, D-8034 Germering

Satz und Druck: Walter Biering GmbH, Grafisscher Betrieb, D-8000 München 45

Offizielles Organ

der Deutschen Gesellschaft für Hämatologie und Onkologie

und der

Österreichischen Gesellschaft für Hämatologie und Onkologie

Offizielles Organ

der österreichischen Krebsgesellschaft - Krebsliga 